\title{
A New Drug Delivery System Using Plasma-Irradiated Polysaccharide
}

\author{
Yukinori Yamauchi $^{1}$, Masayuki Kuzuya ${ }^{2}$, Yasushi Sasai $^{3}$, and Shin-ichi Kondo ${ }^{3}$ \\ ${ }^{1}$ Department of Pharmaceutical Physical Chemistry, \\ College of Pharmaceutical Sciences, Matsuyama University, \\ 4-2 Bunkyo-cho, Matsuyama, Ehime 790-8578, Japan \\ ${ }^{2}$ Department of Health \& Welfare, Chubu Gakuin University, \\ 2-1, Kirigaoka, Seki-shi, Gifu, 501-3993, Japan \\ ${ }^{3}$ Laboratory of Pharmaceutical Physical Chemistry, \\ Gifu Pharmaceutical University, 1-25-4 Daigaku-Nishi, Gifu 501-1196, Japan
}

Keywords: Oxygen plasma irradiation, drug delivery system, cellulose derivatives

\section{Introduction}

Over the past few decades, significant medical advances have been made in the area of drug delivery with the development of controlled release dosage forms [1]. The purpose of the controlled release systems is to maintain drug concentration in the blood or in target tissues at a desired value as long as possible. To reduce the frequency of administration and to improve patient compliance, a sustained-release formulation of various drugs is desirable.

Over the years, we have been working on the development of plasma-assisted preparation of multi-layered tablets applicable to oral drug delivery system (DDS) for sustained- and delayed-release [2-4]. In previous papers, we reported the preparation of sustained release system by oxygen plasma-irradiation to the double-compressed tablet having a mixture of plasma-crosslinkable polystyrene (PSt) and plasma-degradable polyoxymethylene (POM) powders as a wall material [5]. When oxygen plasma was irradiated to the outermost layer of the tablet, POM could be selectively eliminated and simultaneously PSt undergoes the rapid crosslink reaction to result in the formation of the porous outer layer of the tablet. As a result, the drugs could be released from the tablet through the resulting micropores.

In this paper, we used hydroxypropyl methylcellulose acetate succinate (HPMCAS) and hydroxypropyl methyl cellulose phthalate
(HPMCP), widely used pharmaceutical aids as an enteric coating agent, for the outer layer of a double compressed tablet containing theophylline. We studied the applicability of the polymers as the outer layer to this DDS preparation.

\section{Experimental}

\subsection{Materials}

HPMCAS and HPMCP were kindly supplied by Shin-Etsu Chemical Co. (Tokyo, Japan). Lactose monohydrate and myo-Inositol were purchased from Wako Pure Chemical Industry Co. (Tokyo, Japan). All other reagents used were of analytical grade.

\subsection{Tablet Preparation and Plasma-Irradiation}

Tested tablets were obtained by compressing powder mixtures of (A) HPMCAS and myo-inositol or (B) HPMCP and lactose into a flat-faced tablet $(13 \mathrm{~mm} \phi)$ at a pressure of 200 $\mathrm{kg} / \mathrm{cm}^{2}$ for $30 \mathrm{~s}$. The double-compressed tablets were obtained as follows: a flat-faced core theophylline tablet $(50 \mathrm{mg}, 10 \mathrm{~mm} \phi)$ was first prepared at a pressure of $40 \mathrm{~kg} / \mathrm{cm}^{2}$ for $10 \mathrm{~s}$, and then the core tablet was placed onto half of the prescribed amount of powdered mixture $(50 \mathrm{mg})$ as a wall material in a tablet die. After the rest of the powdered mixture $(50 \mathrm{mg})$ was placed on the core tablet, the whole was compressed at pressure of $200 \mathrm{~kg} / \mathrm{cm}^{2}$ for $30 \mathrm{~s}$. All flat-faced tablets were prepared using a hand press instrument in a tablet die (SE work press and SE 
dice, Okada seiko CO., Japan).

A schematic representation of the apparatus for plasma-irradiation is shown in Fig. 2, and this apparatus is essentially the same as reported earlier [2-5].

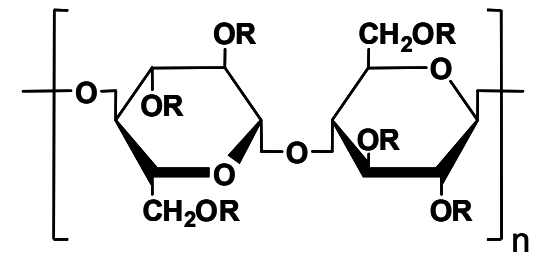

Hydroxypropylmethylcellulose acetate succinate (HPMCAS) $\mathrm{R}=-\mathrm{H},-\mathrm{CH}_{3},-\mathrm{CH}_{2} \mathrm{CH}\left(\mathrm{CH}_{3}\right) \mathrm{OH},-\mathrm{COCH}_{3}$, or $-\mathrm{COCH}_{2} \mathrm{CH}_{2} \mathrm{COOH}$ Hydroxypropylmethylcellulose phthalate (HPMCP) $\mathrm{R}=-\mathrm{H},-\mathrm{CH}_{3},-\mathrm{CH}_{2} \mathrm{CH}\left(\mathrm{CH}_{3}\right) \mathrm{OH}$, or $-\mathrm{COC}_{6} \mathrm{H}_{4} \mathrm{COOH}$

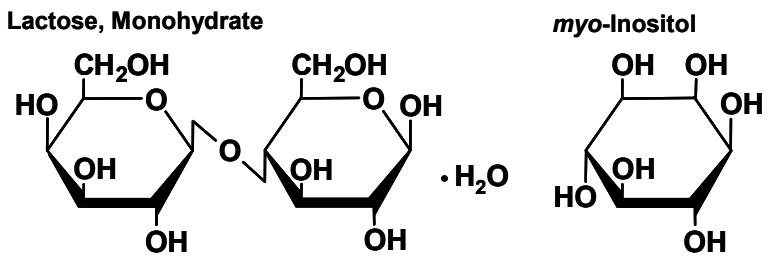

Fig. 1. Chemical structures of HPMCAS, HPMCP, Lactose and myo-Inositol.

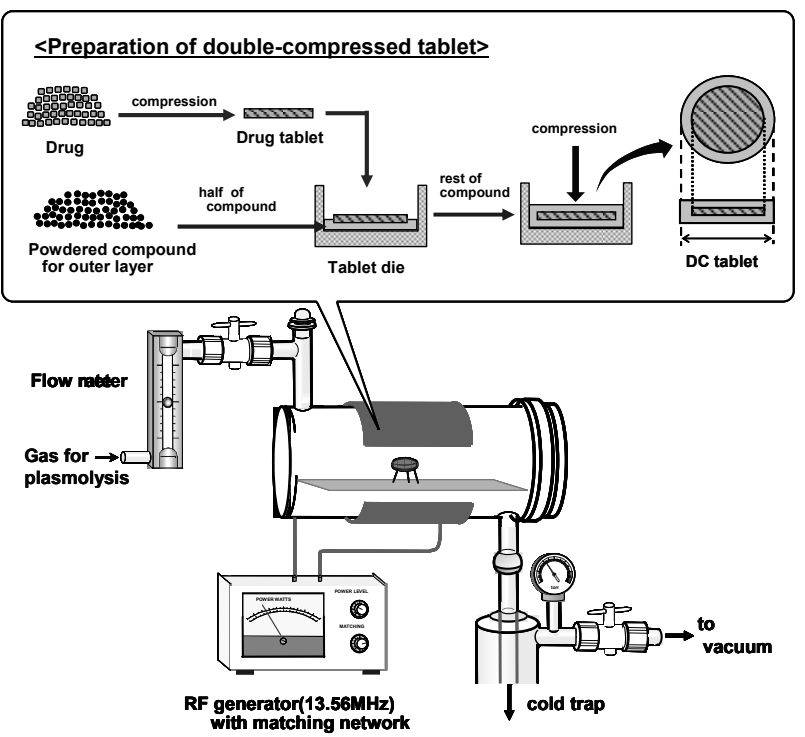

Fig. 2. Schematics for preparation of doublecompressed tablets including the experimental set-up for plasma-irradiation.

The plasma state was generated by use of capacitively coupled parallel-plate radio frequency discharges at $13.56 \mathrm{MHz}$ with supplied power of $50 \mathrm{~W}$. Flow volume $(50 \mathrm{~mL} / \mathrm{min})$ and pressure of argon or oxygen gas $(66.6 \mathrm{~Pa})$ for plasmolysis were controlled by changing evacuating speed. The sample tablets were placed on a glass-tripod in a reaction chamber (240 $\mathrm{mm}$ long, $150 \mathrm{~mm} \phi)$ to ensure homogeneous exposure to plasma gas.
Degradation rate of sample tablets induced by plasma-irradiation was carried out according to the method similar to the above, and determined by measurement of the weight loss at various stages of plasma duration.

\subsection{Scanning Electron Microscope (SEM)}

The microscopic changes in the surface of the plasma-irradiated tablets were photographed by SEM (S-3400N, Hitachi, Japan) with an accelerating voltage of $5 \mathrm{kV}$ and magnification of $\mathrm{x} 1,000$. Prior to the analyses, the samples were sputtered with a thin film of gold.

\subsection{In Vitro Release Studies}

The in vitro dissolution studies were carried out using a dissolution test apparatus with an autosampling apparatus (Toyama Sangyo Co., Osaka, Japan) at $100 \mathrm{rpm}$. The dissolution medium consisted of the $0.1 \mathrm{M}$ phosphate buffer $\mathrm{pH} 7.4(900 \mathrm{~mL})$, maintained at $37 \mathrm{C} \pm 0.5^{\circ} \mathrm{C}$. The drug release at different time intervals was measured by a Shimadzu UV-1800 spectrometer (Shimadzu Inc., Kyoto, Japan) at $270 \mathrm{~nm}$ using UV system ver.3.2 software (Toyama Sangyo Co., Osaka, Japan). It was made clear that none of the ingredients used in the formulations interfered with the assay. The release studies were conducted in triplicate, and the mean values were plotted versus time.

\section{Results and Discussion}

3.1 Degradation Property of HPMCAS, HPMCP, Lactose and myo-Inositol by Plasma-Irradiation

The degradation property of materials by plasma irradiation is known to be dependent on the plasma reaction apparatus and shape of the samples. In order to understand the nature of plasma-irradiation effect on degradation of HPMCAS, HPMCP, lactose and myo-inositol, we therefore studied the plasma degradation property of samples focusing on the changes in the weight loss of the directly-compressed tablets by plasma irradiation using the apparatus shown in Fig. 2. Figure 3 illustrates changes in the weight loss of directly-compressed tablets according to the argon and oxygen plasma irradiation time in comparison with the results obtained with typical cross-linkable PSt and degradable cellulose and POM.

It is seen that degradation for each sample is linearly proportional to the plasma duration time within the time studied. The degradation rates 
of HPMCAS (Ar; $0.14 \mathrm{mg} \cdot \mathrm{cm}^{-2} \cdot \mathrm{min}^{-1}, \mathrm{O}_{2} ; 0.33$ $\mathrm{mg} \cdot \mathrm{cm}^{-2} \cdot \mathrm{min}^{-1}$ ) and HPMCP (Ar; $0.11 \mathrm{mg} \cdot \mathrm{cm}^{-2}$. $\left.\mathrm{min}^{-1}, \mathrm{O}_{2} ; 0.34 \mathrm{mg} \cdot \mathrm{cm}^{-2} \cdot \mathrm{min}^{-1}\right)$ are lower than those of cellulose $\left(\mathrm{Ar} ; 0.27 \mathrm{mg} \cdot \mathrm{cm}^{-2} \cdot \mathrm{min}^{-1}, \mathrm{O}_{2}\right.$; $\left.0.57 \mathrm{mg} \cdot \mathrm{cm}^{-2} \cdot \mathrm{min}^{-1}\right)$ in both argon and oxygen plasma-irradiations. This can be explained in terms of the fact that a carboxyethyl group in HPMCAS and a carboxybenzoyl group in HPMCP have a great feasibility of the surface cross-link reaction which are faster in rate than the reaction of plasma-activated oxygen species with the polymers in oxygen plasma-irradiation. Whereas the difference in the degradation property of lactose and myo-inositol between argon and oxygen plasma irradiation is large compared with that of other polymers, suggesting that the degradation reaction occurs more easily. Based on the fact that the value of weight loss increases as the plasma duration increases, it seems apparent that plasma degradable lactose and myo-inositol could be selectively eliminated to result in the formation of the porous outer layer of the tablet. Then, theophylline could be released from the tablet through the resulting micropores.
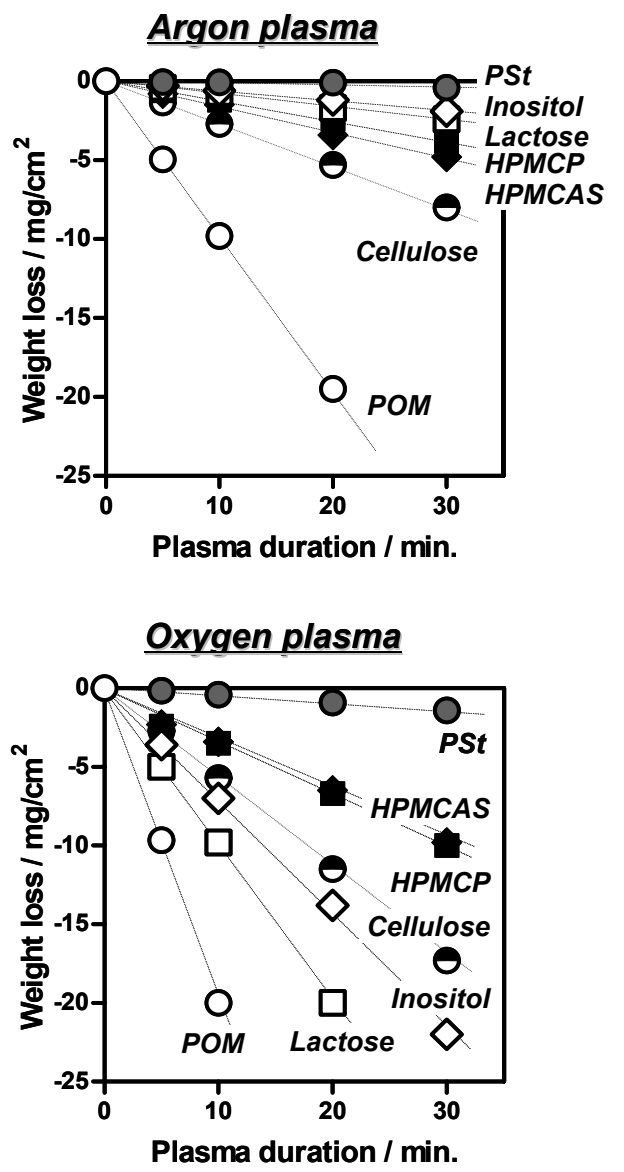

Fig. 3. Effect of plasma-irradiation on the degradation of flat-faced compressed tablets consisted of various compounds.

Plasma condition: $50 \mathrm{~W}, 50 \mathrm{~mL} / \mathrm{min}, 66.6 \mathrm{~Pa}$.
3.2 Theophylline Release from Plasma-Irradiated Double-Compressed Tablets

The two types of double-compressed tablets containing theophylline as a core material $(50 \mathrm{mg}$, $10 \mathrm{~mm}$ in diameter) and mixture of (A) HPMCAS and myo-inositol or (B) HPMCP and lactose in $7: 3$ by weight as a wall material (mixture $100 \mathrm{mg}$, $13 \mathrm{~mm}$ in diameter) were prepared. Dissolution experiments of the plasma-treated and untreated tablets were carried out using the rotating basket apparatus method. Figure 4 illustrates the dependence of the release property of theophylline in a buffer solution at $\mathrm{pH} 7.4$ from the tablets on the plasma irradiation time. As is clear in Fig. 4, plasma-untreated (blank) and 2-min plasma-treated tablets showed quick disintegration and rapid release of theophylline due to combined effects of water absorption and swelling by the formulation. On the other hand, all the tablets plasma-irradiated for more than 4 min have clearly shown to convert into a sustained-release tablet and it takes as long as 12 $-24 \mathrm{~h}$ for the theophylline release to complete in $\mathrm{pH} 7.4$ buffer solution. It was also shown that excessive plasma exposure for more than $10 \mathrm{~min}$ increased in drug release rate.

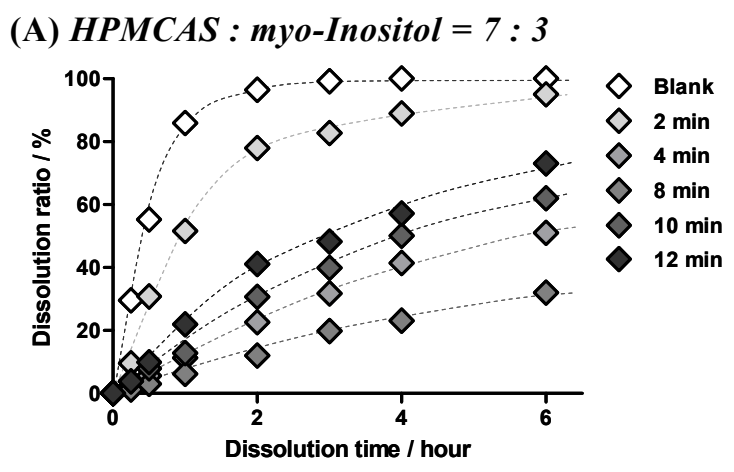

(B) HPMCP : Lactose $=7: 3$

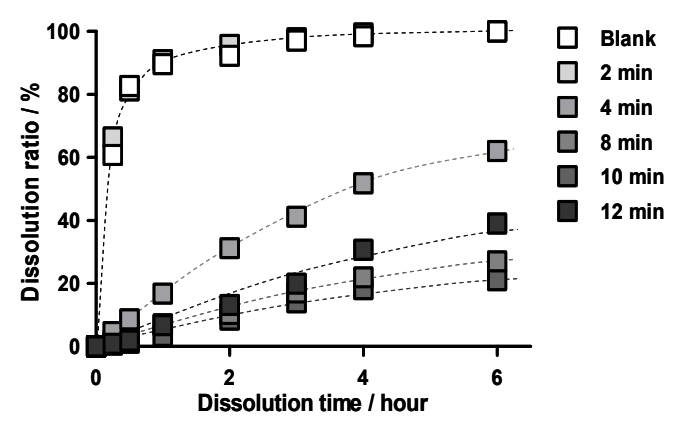

Fig 4. Effect of plasma durations on Theophylline release properties in $\mathrm{pH} 7.4$ buffer solution from plasma-irradiated double-compressed tablets using powdered mixtures of (A) HPMCAS-myo-Inositol and (B) HPMCP-Lactose as outer layer.

Plasma conditions: $\mathrm{O}_{2} 50 \mathrm{~mL} / \mathrm{min}, 66.6 \mathrm{~Pa}$. 
Thus, when the combinations of HPMCASmyo-Inositol (7:3) or HPMCP-Lactose (7:3) are used as a wall material of the double-compressed tablet, the plasma-irradiated tablets showed a very slow release pattern with plasma duration. It could be considered that the present tablets of HPMCAS and HPMCP are apparently applicable to controlled-release systems having a desired release pattern by selecting the plasma operational conditions.

3.3 Observation of Surfaces of Outer Layers of the double-compressed tablets

In order to understand the factor to control the nature of drug release pattern, the progressive changes in the surface morphology of the plasma-irradiated tablets $(x$ 1,000) were monitored by SEM observations.

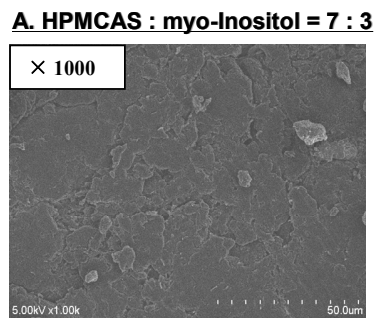

(a) Blank

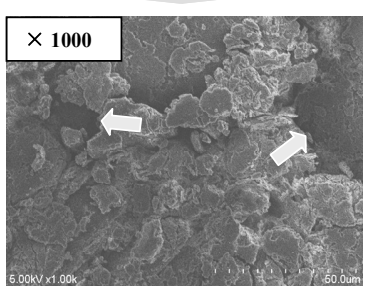

(b) 4 min

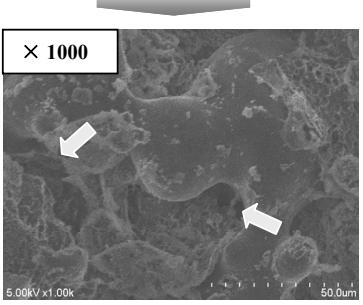

(c) $10 \mathrm{~min}$

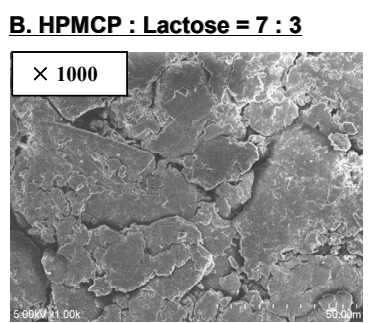

( a ) Blank

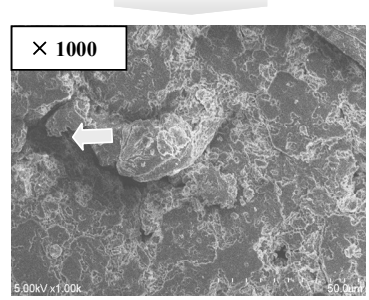

(b) $4 \mathrm{~min}$

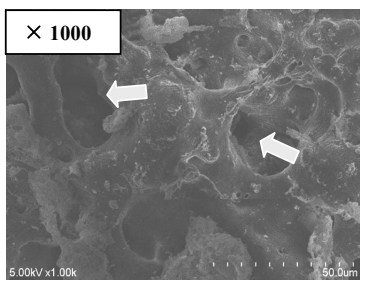

(c) $10 \mathrm{~min}$
Fig. 5. SEM images of double-compressed tablets before and after plasma-irradiation for various duration.

Plasma conditions: $\mathrm{O}_{2} 50 \mathrm{~mL} / \mathrm{min}, 66.6 \mathrm{~Pa}$.

Figure 5 presents several SEM images on the surface of double-compressed tablets of HPMCAS-myo-Inositol and HPMCP-Lactose, before and after plasma-irradiation for 4 and 10 min. The plasma-irradiated tablets showed the progress of surface softening, as the plasma duration increased. It is apparent that plasma irradiation caused cross-link reaction of HPMCAS and HPMCP and changed the tablet surface into a web-linked network, resulting in the formation of film-like surface by clogging the space/crack existing at particle-particle interfaces. Furthermore it is clearly seen that plasmairradiation generate micropores with an approximate size of 2-10 um, resulting in the formation of the porous outer layer. It should be considered that the formation of various sizes of micropores was caused by the oxidative decomposition and/or vaporization of myoinositol and lactose.

\section{Conclusion}

In this study, we attempted to control the drug release from a double-compressed tablet containing theophylline core with outer layer consisted of HPMCAS-myo-Inositol and HPMCP-Lactose by plasma-irradiation.

By use of difference in degradation properties of organic compounds, the oxygen plasmairradiation varied outer layer so as to cause release of drugs at different rates, depending on conditions chosen for plasma operation. From the SEM analyses, it was concluded that concurrent occurrence of cross-link reaction and the micropores formation on outer layer converted rapid-release system into sustained-release system.

As described, it was shown that new examples that cellulose derivatives widely used as pharmaceutical aids would be also applied to a outer layer polymer of the double-compressed tablet to fabricate a system for targeted release of various kinds of drugs in the lower sections of the small or large intestine by making use of plasma techniques.

\section{References}

1. G.M. Khan, The Sciences, 1(5), (2001), 350. 2. M. Kuzuya, Y. Sasai, S. Kondo, Y. Yamauchi, Curr. Drug Discov. Tech., 6, (2009) 135.

3. M. Kuzuya, Y. Sasai, Y. Yamauchi, S. Kondo, Industrial Plasma Technology: Applications from Environmental to Energy Technologies, WILEYVCH Verlag GmbH \& Co,in part, (2010) pp.113. 4. Y. Sasai, S. Kondo, Y. Yamauchi, M. Kuzuya, Biomecdical Engineering, INTECH, (2011) pp101.

5. M. Kuzuya, A. Noguchi, H. Ito, M. Ishikawa, Drug Delivery System, 6, (1991), 119. 